\title{
Logarithmically Slow Expansion of Hot Bubbles in Gases
}

\author{
Baruch Meerson and Pavel V. Sasorovt \\ Racah Institute of Physics, Hebrew University of Jerusalem, Jerusalem 91904, Israel \\ Ken Sekimoto \\ Yukawa Institute for Theoretical Physics, Kyoto University, Kyoto 606-8502, Japan
}

\begin{abstract}
We report logarithmically slow expansion of hot bubbles in gases in the process of cooling. A model problem first solved, when the temperature has compact support. Then temperature profile decaying exponentially at large distances is considered. The periphery of the bubble is shown to remain essentially static ("glassy") in the process of cooling until it is taken over by a logarithmically slowly expanding "core". An analytical solution to the problem is obtained by matched asymptotic expansion. This problem gives an example of how logarithmic corrections enter dynamic scaling.

PACS numbers: 47.54.+r, 47.40.Dc, 02.30.Jr
\end{abstract}

Dynamic scaling behavior of extended nonlinear systems out of equilibrium have attracted much attention in different areas of physics [1. In continuum models dynamic scaling is intimately related to self-similar asymptotics of nonlinear partial differential equations [2. Sometimes logarithmic corrections enter dynamic scaling laws [1]. No general scenario for their appearance is known. One can say from experience that they appear in marginal cases, dividing (in an appropriate functional space) regimes with qualitatively different behavior. The aim of this work is to investigate one particular setting, of general interest, where logarithmic corrections to scaling appear somewhat unexpectedly: cooling dynamics of hot bubbles in gases.

Heat transfer in gases, strongly heated locally, looks quite differently from the simple picture provided by the linear heat diffusion equation. The difference is mainly due to the small-Mach-number gas flow that develops (even at zero gravity) owing to intrinsic pressure gradients. This conductive cooling flow (CCF) brings in cold gas from the periphery and strongly modifies the cooling dynamics. Some aspects of CCFs have been studied experimentally and theoretically, mainly in the context of the late stage of strong explosions [3 5 . In this Letter we report a new, striking feature of a CCF. We find that, if the initial temperature profile decays rapidly enough at large distances [like $\exp (-k|x|), k>0$ ], the hot bubble, while cooling down significantly, expands logarithmically slowly.

Starting from the continuity, momentum and energy equations for an inviscous ideal gas at zero gravity, and employing the small-Mach-number expansion, one arrives at the following nonlinear equation for the scaled gas temperature [3]:

$$
\partial_{t} T=T^{2} \partial_{x}\left(T^{\nu-1} \partial_{x} T\right)
$$

where indices $t$ and $x$ stand for partial derivatives (a slab geometry is assumed), and $\nu$ is the exponent in the power-law temperature dependence of the heat conductivity of the gas [6].
The scaled gas pressure stays constant (and equal to unity) in this approximation, so the scaled gas density is simply $\rho(x, t)=T^{-1}(x, t)$, while the gas velocity is $v(x, t)=T^{\nu} \partial_{x} T$ [3]. Therefore, once solving Eq. (11) for the temperature, one can easily find all other variables.

Eq. (1) has a multitude of similarity solutions:

$$
T_{\beta}(x, t)=t^{\frac{2 \beta-1}{\nu+1}} \theta\left(x / t^{\beta}\right),
$$

where $\beta$ is an arbitrary parameter. Therefore, an interesting selection problem appears, like in many other situations in nonlinear dynamics of extended systems [2, [7.

Eq. (11) has appeared in the context of cooling of the "fireball" produced by a strong local explosion in a gas [3 5]. An explosion involves energy release on a time scale short compared to the characteristic acoustic time. In this case the preceding rapid stage of the dynamics produces an inverse power-law dependence of the gas temperature on the distance from the explosion site [8]. It has been shown 3., 4 that the exponent of this power law uniquely selects the scaling exponent $\beta$. As the result, the fireball expansion exhibits a power law in time.

A different type of local gas heating occurs when the time scale of the energy release is long compared to the acoustic time, but still short compared to the cooling time. In this case the initial temperature profile is more localized, as it reflects the spatial structure of the heating agent (for example, the radial intensity of laser beam). This regime will be in the focus of this Letter. We will see that there is no self-similar asymptotics to this problem. Instead, the solution approaches, at long times, a "quasisimilarity" asymptotics with logarithmic corrections to scaling.

Consider first a model problem when the initial temperature profile has compact support: $T(x, 0)=T_{0}(x)>$ 0 at $x \in[-L, L]$, and zero elsewhere. We will limit ourselves to a temperature-independent heat conductivity, $\nu=0$. Despite this choice, the nonlinearity of Eq. (11) persists. Assume symmetry with respect to $x=0$ and impose the Neumann boundary conditions: $\partial_{x} T(0, t)=$ 
$\partial_{x} T(L, t)=0$. A local analysis of Eq. (11) near the edge of support of its solution $T(x, t)$ shows that the support remains compact and unchanged for $t>0$. Therefore, this model problem exhibits a complete localization. What is the late-time behavior of the temperature? The constancy of support immediately selects $\beta=0$, so the similarity ansatz becomes $T_{0}(x, t)=t^{-1} \theta(x)$. Then Eq. (1) yields $\theta(x)=\left(a^{2} / 2\right) \cos ^{2}(x / a)$ for $x \in[-L, L], \theta(x)=0$ elsewhere, and $a=\pi L / 2$. This simple similarity solution describes cooling of the hot bubble (and filling it with the dense gas) without any change in the bubble size.

Remarkably, $T_{0}(x, t)$ represents a long-time asymptotics for any initial condition that has compact support $[-L, L]$ and obeys the Neumann boundary conditions. We will show here only that this solution is linearly stable with respect to small perturbations, and find the spectrum of the linearized problem. Introduce new variables: $u=t T(x, t)$ and $\tau=\ln t$. Eq. (11) assumes the form

$$
\partial_{\tau} u=u-\left(\partial_{x} u\right)^{2}+u \partial_{x x} u
$$

while the similarity solution $T_{0}(x, t)$ becomes steadystate solution $\theta(x)$. Introducing a small correction $v(x, \tau)$ to this solution and linearizing Eq. (3), we obtain $\partial_{\tau} v=\hat{L}(\xi) v$, where

$$
\hat{L}(\xi)=(1 / 2) \cos ^{2} \xi \partial_{\xi \xi}+\sin 2 \xi \partial_{\xi}+2 \sin ^{2} \xi
$$

and $\xi=x / a$. We look for the eigenfunctions in the form of $v(\xi, \tau)=e^{\gamma \tau} \psi_{\gamma}(\xi)$. The general solution of the resulting ordinary differential equation is

$$
\begin{aligned}
& \psi_{\gamma}(\xi)=C_{1} \cos ^{2} \xi_{2} F_{1}\left(a_{-}, a_{+}, 1 / 2,-\tan ^{2} \xi\right) \\
& \quad+C_{2} \cos \xi \sin \xi_{2} F_{1}\left(b_{-}, b_{+}, 3 / 2,-\tan ^{2} \xi\right)
\end{aligned}
$$

where ${ }_{2} F_{1}$ is the hypergeometric function, $C_{1}$ and $C_{2}$ are arbitrary constants, $a_{ \pm}=\left[1 \pm(8 \gamma+9)^{1 / 2}\right] / 4$ and $b_{ \pm}=\left[3 \pm(8 \gamma+9)^{1 / 2}\right] / 4$. Requiring that the perturbation remains small compared to the unperturbed solution [and hence vanishes like $(\pi / 2-\xi)^{2}$ or faster at $\xi \rightarrow \pi / 2$ ], we find the (continuous) spectrum of the linearized problem: $-\infty<\gamma \leq-1$. This result proves linear stability of the similarity solution $T_{0}(x, t)$. Notice the presence of gap between the upper edge of the spectrum $\gamma=-1$ and stability border $\gamma=0$. Going back to physical variables, we find that small temperature perturbations around the similarity solution exhibit a power law decay $t^{\gamma-1}$.

At this stage we notice that $\beta=0$ is a marginal case dividing two qualitatively different types of dynamics as described by the family of similarity solutions (2). Indeed, solutions with $\beta>0$ describe power-law expansions [3 5], while solutions with $\beta<0$ correspond to power-law shrinkings [9]. One can expect logarithmic corrections to appear "on the background" of the special case $\beta=0$, when the initial condition does not have compact support, but decays rapidly at large distances. Therefore, we assume that the initial temperature profile of the bubble is symmetric with respect to $x=0$ and decays exponentially at large distances [10]. We will continue using the new variables and require $u(x, 0) \rightarrow c \exp (-k|x|)$ at $|x| \rightarrow \infty$, where $k$ and $c$ are positive constants. One can always put $k=1$ 11]. We will be interested in a long-time asymptotics of the solution: $\tau \gg 1$. Our first important observation is that $u(x, \tau)=c \exp (\tau-x)$ is an exact solution of Eq. (3). This traveling wave solution with a unit speed corresponds to a steady-state solution $T(x)=c \exp (-x)$ in physical variables, and it represents the correct asymptotics of the solution to our problem at $x \rightarrow+\infty$. What about the bubble "core"? We will show that it can be described, at $\tau \gg 1$, by a "quasi-similarity" solution plus small corrections:

$$
u(x, \tau)=u_{0}(x, \tau)+u_{1}(x, \tau)+\ldots,
$$

where

$$
u_{0}(x, \tau)=\frac{a^{2}(\tau)}{2} \cos ^{2} \frac{x}{a(\tau)}
$$

and $\ldots \ll u_{1} \ll u_{0}$. One of our goals is to find an asymptotic expansion for $a(\tau)$.

The leading term of $a(\tau)$ can be guessed immediately. Indeed, expansion of $u_{0}(x, \tau)$ in powers of $x-\pi a(\tau) / 2$ near the point $x=\pi a(\tau) / 2$ begins with the term $(1 / 2)(x-\pi a(\tau) / 2)^{2}$. This is a wave traveling with speed $\pi \dot{a} / 2$ along the $x$-axis! Therefore, it is natural to look for a general traveling wave solution $v(x, \tau)=V(x-\tau)$ of Eq. (3) with a unit speed and require that it behaves like $(z+\text { const })^{2} / 2$ at $z \rightarrow-\infty$ and like $c \exp (-z)$ at $z \rightarrow+\infty$, where $z=x-\tau$. If such a solution exists, we can match it with the leading term of the quasi-similarity solution (7) in the region $1 \ll-(x-\pi a / 2) \ll a, 1 \ll-z$, once

$$
a(\tau)=2 \tau / \pi .
$$

Eqs. (7) and (8) have important implications. First, the temperature scaling with physical time $t$ at the bubble center acquires a logarithmic correction. Second, the bubble core expands logarithmically slowly. We will show in the following that these are indeed correct results, calculate the subleading and sub-subleading terms for $a(\tau)$, and find other attributes of asymptotic solution.

The general traveling wave solution of Eq. (3), $V(z)$, obeys the second-order equation

$$
-V_{z}=V-V_{z}^{2}+V V_{z z}
$$

that is soluble analytically. One integration yields

$$
V^{-1}(d V / d z)=-1-W\left(-\exp \left(-1-V^{-1}\right)\right),
$$

where $W(\eta)$ is the product log function defined as the solution of equation $W e^{W}=\eta$ (see, e.g., Ref. [12, p. 751). The arbitrary constant in Eq. (10) has been chosen 
to satisfy the required asymptotic behavior $V(z) \rightarrow(z+$ const $)^{2} / 2$ at $z \rightarrow-\infty$. Notice that, as $V>0$ and $V_{z}<0$, we should work with the negative branch of the product log function: $\eta<0$ and $W(\eta)<0$.

Integrating Eq. 10], we obtain the traveling wave solution in an implicit form:

$$
J(V)+z+C=0
$$

where

$$
J(V)=\int_{1}^{U(V)} \frac{d \zeta}{1-\zeta-e^{-\zeta}}
$$

$U(V)=-\ln \left[-W\left(-\exp \left(-1-V^{-1}\right)\right)\right]$ and $C$ is an arbitrary constant.

To understand the asymptotic behavior of this solution at $z \rightarrow-\infty$ and $z \rightarrow+\infty$, we need to know the asymptotics of $J(V)$. After some algebra we obtain

$$
J(V)=\left\{\begin{array}{l}
\ln V+\Delta_{1}+\mathcal{O}(V), \\
(2 V)^{1 / 2}+\frac{1}{3} \ln V+\Delta_{2}+\mathcal{O}\left(V^{-1 / 2}\right),
\end{array}\right.
$$

at $V \rightarrow+0$ and $V \rightarrow+\infty$, respectively. Here

$$
\Delta_{1}=\int_{1}^{\infty} \frac{\left(1-e^{-\zeta}\right) d \zeta}{\zeta\left(1-\zeta-e^{-\zeta}\right)}=-1.46074400 \ldots,
$$

$\Delta_{2}=-2-(1 / 3) \ln (2 e)-\Delta_{3}$, and

$\Delta_{3}=\int_{0}^{1}\left(\frac{1}{1-\zeta-e^{-\zeta}}+\frac{2}{\zeta^{2}}+\frac{2}{3 \zeta}\right) d \zeta=-0.05361892 \ldots$

Using Eqs. (11) and (13) we obtain:

$$
V(z)=e^{-z-C-\Delta_{1}}+\mathcal{O}\left(e^{-2 z}\right) \quad \text { at } \quad z \rightarrow+\infty
$$

and

$$
\begin{aligned}
V(z)= & (1 / 2)\left(z+C+\Delta_{2}\right)^{2} \\
& \left.+(2 / 3)\left(z+C+\Delta_{2}\right) \ln \left(\left|z+C+\Delta_{2}\right|\right) / \sqrt{2}\right) \\
& +\mathcal{O}\left(\ln ^{2}\left|z+C+\Delta_{2}\right|\right) \quad \text { at } z \rightarrow-\infty .
\end{aligned}
$$

The required asymptotic behavior $V \rightarrow c \exp (-z)$ at $z \rightarrow+\infty$ selects $C=-\Delta_{1}-\ln c$, so the traveling wave solution (11) is now fully determined. After some rearrangement, we rewrite the asymptotics (14) and (15) as

$$
V(z)=c e^{-z}+\mathcal{O}\left(e^{-2 z}\right) \quad \text { at } \quad z \rightarrow+\infty,
$$

and

$$
\begin{aligned}
V(z)= & (1 / 2)(z-\Delta)^{2}+(2 / 3)(z-\Delta) \ln |z-\Delta| \\
& +\mathcal{O}\left(\ln ^{2}|z|\right) \quad \text { at } \quad z \rightarrow-\infty,
\end{aligned}
$$

where $\Delta=\Delta_{1}-\Delta_{2}+(1 / 3) \ln 2+\ln c$.

The leading term in Eq. (16) corresponds to a steadystate solution in the physical variables, while the subleading term is exponentially small with respect to the leading one. This essentially static ("glassy") behavior of the solution at large distances reflects effective diffusion choking at small temperatures.

Now let us return to the bubble core description, Eq. (7). Our basic assumption here (supported by the results) is that, in the asymptotic stage $\tau \gg 1$, the terms $u_{0}, u_{1}, \ldots$ depend on time only through the time dependences of $a$, of $a$ and $\dot{a}$, of $a, \dot{a}, \ldots$, respectively. The small parameter of this expansion is $\dot{a} / a$. In the zeroth approximation of this perturbation scheme, $u_{0}$ obeys Eq. (3) without the time derivative term. In the first approximation we obtain the following linear equation:

$$
\hat{L} u_{1}(\xi)=a \dot{a} \cos ^{2} \xi(1+\xi \tan \xi),
$$

where we have again used $\xi=x / a$.

The zero modes of the operator $\hat{L}$ are $\Upsilon(\xi)=\cos ^{2} \xi+$ $\xi \cos \xi \sin \xi$ and $\Phi(\xi)=\sin \xi \cos \xi$. Looking for the general solution of Eq. (18) in the form of $u_{1}=$ $C_{1}(\xi) \Upsilon(\xi)+C_{2}(\xi) \Phi(\xi)$ and defining $a(\tau)$ by the condition $u(0, \tau)=a^{2}(\tau) / 2$, we arrive at

$$
\begin{aligned}
u_{1}(\xi)= & -(2 / 3) a \dot{a} \cos ^{2} \xi[(\xi \tan \xi-1) \ln \cos \xi \\
& \left.+2 \ln (2 / e) \xi \tan \xi+\tan \xi \operatorname{Im}\left[\operatorname{Li}_{2}\left(-e^{2 i \xi}\right)\right]\right]
\end{aligned}
$$

where $\operatorname{Li}_{2}(x)=\sum_{k=1}^{\infty} k^{-2} x^{k}$ is the dilogarithm (see, e.g., Ref. [12], p. 743). In the vicinity of $\xi=\pi / 2$

$$
u_{1}=\frac{\pi}{3} a \dot{a} \tilde{\xi} \ln \frac{4|\tilde{\xi}|}{e^{2}}+a \dot{a} \mathcal{O}\left(|\tilde{\xi}|^{3} \ln |\tilde{\xi}|\right)
$$

where $\tilde{\xi}=\xi-\pi / 2$. Too close to $\xi=\pi / 2$ the correction $u_{1}$ and its derivatives become larger than the zero-order solution $u_{0}$ and its corresponding derivatives, so the perturbation procedure breaks down. Therefore, the bubble core solution [Eqs. (6), (7) and (19)] should be matched with the traveling wave solution [Eq. (11)] in the region where $|x-\pi a / 2|$ is small enough (so that the leading term of the asymptotics of $u_{0}$ is much larger than the subleading terms) but, on the other hand, large enough (so that $u_{1}$ is small compared to $u_{0}$ ). Working in this region and collecting the leading contributions from $u_{0}$ and $u_{1}$, we obtain after some rearrangement:

$$
\begin{aligned}
u= & \frac{1}{2} \tilde{x}^{2}+\frac{\pi}{3} \dot{a} \tilde{x} \ln |\tilde{x}|+\frac{1}{a^{2}} \mathcal{O}\left(\tilde{x}^{4}\right)+\frac{\dot{a}}{a^{2}} \mathcal{O}\left(|\tilde{x}|^{3} \ln |\tilde{x}|\right) \\
& +\mathcal{O}\left(\dot{a}^{2} \ln ^{2} a\right) \mathcal{O}(\ln |\tilde{x}|)+\ldots,
\end{aligned}
$$

where $\tilde{x}=x-(\pi / 2) a-(\pi / 3) \dot{a} \ln \left(e^{2} a / 4\right)$.

Now we can perform the matching procedure. We require that the $z \rightarrow-\infty$ asymptotics of the traveling wave solution, Eq. (17), coincide with the asymptotics (21) of the bubble core solution. This yields 


$$
a(\tau)=\frac{2}{\pi k}\left[\tau-\frac{2}{3} \ln \frac{\tau}{4 \pi}+B+\ln c k^{2}+o(1)\right],
$$

where $B=1+\Delta_{1}+\Delta_{3}=-0.514362926 \ldots, o(1)$ denotes terms that vanish as $\tau \rightarrow \infty$ and we have restored the $k$-dependence [11]. We see that the leading term in $a(\tau)$ is logarithmic in physical time $t$, and it coincides with Eq. (3). The subleading term behaves like $\ln \tau \sim \ln \ln t$, while the sub-subleading term is constant.

The matching region is determined by the requirements that the subleading term in Eq. (21) is much less than the leading term, but much greater than the rest of terms. These yield an approximate condition $\ln ^{2} a \ll-x+\frac{\pi}{2} a \ll a^{2 / 3}$, so that the matching region expands as $\tau \rightarrow \infty$.

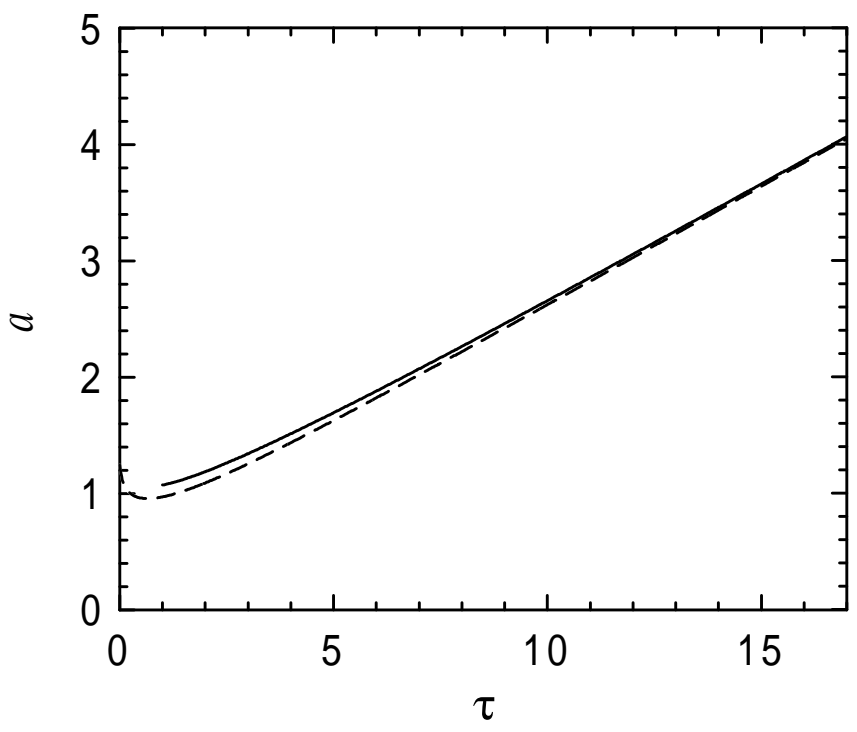

FIG. 1. The amplitude $a$ versus the new time $\tau=\ln t$ found numerically (dashed line) and analytically (solid line). The parameters are described in the text.

We compared the asymptotic solution with numerical simulations. Finding logarithmic corrections numerically usually requires going to very long times. Instead, we directly solved Eq. (3) in the new variables, which enabled us to reach $\tau \sim 20$, that is $t \sim 5 \cdot 10^{8}$. Eq. (3) was solved on the interval $x \in(0,9)$ subject to the Neumann boundary conditions. The initial condition was $u(x, \tau=0)=2 \exp \left[-3\left(x^{2}+0.1\right)^{1 / 2}\right]$, so that $c=2$ and $k=3$. The system length was large enough for the solution to enter the asymptotic regime before the expanding "core" reaches the boundary $x=9$. Fig. 1 compares $a=(2 u(0, \tau))^{1 / 2}$ found numerically with the prediction of Eq. (22). At long times the agreement is excellent. We also verified other attributes of the asymptotic solution.

In separate simulations that will be presented elsewhere [13], evolution of the same initial condition was investigated in the framework of the full gasdynamic equations. Remarkably, the results essentially coincide, even for moderate Mach numbers, with those obtained with Eq. (1). This shows robustness of the reduced equation in the description of CCFs.

In conclusion, we have shown that hot bubbles in gases expand logarithmically slowly in the process of cooling. By constructing an asymptotic solution, that matches a "quasi-similarity" inner solution and a "glassy" outer solution, we have been able to see how logarithmic corrections enter dynamic scaling.

We are grateful to Y. Kurzweil for help with Fig. 1. This work was partially supported by the COE Visiting Research Scholar Program at YITP and by the Russian Foundation for Basic Research (grant No. 99-01-00123).

* On leave from the Institute of Theoretical and Experimental Physics, Moscow 117259, Russia.

[1] A.J. Bray, Adv. Phys. 43, 357 (1994); A.-L. Barabási and H.E. Stanley, Fractal Concepts in Surface Growth, (Cambridge Univ. Press, Cambridge, 1995); N. Goldenfeld, Lectures on Phase Transitions and the Renormalization Group (Addison-Wesley, 1992).

[2] G.I. Barenblatt, Scaling, Self-similarity, and Intermediate Asymptotics (Cambridge Univ. Press, Cambridge, 1996).

[3] B. Meerson, Phys. Fluids A 1, 887 (1989).

[4] D. Kaganovich, B. Meerson, A. Zigler, C. Cohen and J. Levin, Phys. Plasmas 3, 631 (1996).

[5] A. Glasner, E. Livne and B. Meerson, Phys. Rev. Lett. 78, 2112 (1997).

[6] Alternatively, one obtains a nonlinear diffusion equation for the gas density: $\partial_{t} \rho=\partial_{x}\left(\rho^{-\nu-1} \partial_{x} \rho\right)$, with the effective diffusion coefficient decreasing with $\rho$. This equation appeared in a number of nonlinear diffusion problems where $\rho$ decays at $|x| \rightarrow \infty$ [14. In the hot bubble problem $\rho \rightarrow \infty$ at $|x| \rightarrow \infty$, and this difference results in quite a different dynamics.

[7] M.C. Cross and P.C. Hohenberg, Rev. Mod. Phys. 65, 851 (1993).

[8] Ya. B. Zel'dovich and Yu. P. Raizer, The Physics of Shock Waves and High Temperature Hydrodynamic Phenomena (Academic, New York, 1967).

[9] Shrinking is possible in the problem of heating of a cold "cloud" by a hot and underdense peripheral gas. Some aspects of this regime were investigated in Ref. [14.

[10] In reality, the temperature should approach a finite value at large distances. Correspondingly, the solution we are interested in represents an intermediate asymptotics [2].

[11] If $u(x, \tau)$ is a solution of Eq. (3), then $\lambda^{2} u(x / \lambda, \tau)$ is also a solution for any $\lambda>0$. This property enables one to restore the $k$-dependence in the final results.

[12] S. Wolfram, The Mathematica Book, 3rd edition (Cambridge Univ. Press, Cambridge, 1996).

[13] Y. Kurzweil, E. Livne and B. Meerson (unpublished).

[14] P. Rosenau, Phys. Rev. Lett. 74, 1056 (1995). 